\title{
Approximate Solutions for Conservative Nonlinear Oscillators by He's Homotopy Method
}

\author{
Augusto Beléndez ${ }^{\mathrm{a}}$, Mariela L. Álvarez ${ }^{\mathrm{a}}$, David I. Méndez ${ }^{\mathrm{a}}$, Elena Fernández ${ }^{\mathrm{b}}$, \\ María S. Yebra ${ }^{\mathrm{a}}$, and Tarsicio Beléndez ${ }^{\mathrm{a}}$ \\ a Departamento de Física, Ingeniería de Sistemas y Teoría de la Señal, Universidad de Alicante, \\ Apartado 99, E-03080 Alicante, Spain \\ b Departamento de Óptica, Farmacología y Anatomía, Universidad de Alicante, Apartado 99, \\ E-03080 Alicante, Spain
}

Reprint requests to A. B.; Fax: +34-96-5909750; E-mail: a.belendez@ua.es

Z. Naturforsch. 63a, 529 - 537 (2008); received March 10, 2008

\begin{abstract}
He's homotopy perturbation method is adapted to calculate higher-order approximate periodic solutions of conservative nonlinear oscillators for which the elastic force term is an odd nonlinear function. The method is modified by truncating the infinite series corresponding to the first-order approximate solution before introducing this solution in the second-order linear differential equation, and so on. With carefully constructed iterations, only a few iterations can provide very accurate analytical approximate solutions. An example is presented to illustrate the usefulness and effectiveness of the proposed technique, and comparison of the results obtained using this method with those obtained using other techniques reveals that the former is very effective and convenient.
\end{abstract}

Key words: Nonlinear Oscillators; Approximate Solutions; Homotopy Perturbation Method.

\section{Introduction}

The study of nonlinear problems is of crucial importance in all areas of physics, since most physical phenomena in our world are essentially nonlinear and are described by nonlinear equations. It is very difficult to solve nonlinear problems and, in general, it is often more difficult to get an analytic approximation instead of a numerical one for a given nonlinear problem. There are several methods used to find analytical approximate solutions to nonlinear problems, such as perturbation techniques [1-5], harmonic balancebased methods [5-10] or other techniques [11-19]. Excellent reviews on some asymptotic methods for strongly nonlinear equations can be found in detail in $[20,21]$.

In the present paper we obtain higher-order analytical approximations to the periodic solutions of conservative nonlinear oscillators for which the elastic restoring force is an odd nonlinear function. To do this, we apply He's homotopy perturbation method [2235]. Recently, a considerable amount of attention has been directed towards analytical solutions of nonlinear equations without possible small parameters. To eliminate this disadvantage $\mathrm{He}$ adopted the homotopy technique which is widely applied in differential topo- logy for obtaining an approximate analytical solution of nonlinear differential equations [21]. The homotopy perturbation method, a coupling of the traditional perturbation method and homotopy in topology, deforms continuously a difficult problem to a simple problem which can be solved easily.

We introduce a modification in He's homotopy perturbation method. Applying the standard homotopy perturbation method to nonlinear oscillators for which the restoring force has a nonpolynomial form, an infinite series is obtained for the first analytical approximate solution, and this series must be introduced in the linear differential equation to obtain the secondorder approximate solution. However, since it is difficult to work with an infinite series, we truncate this series before solving the subsequent linear differential equation considering only three harmonics for the second-order approximation, four harmonics for the third-order approximation, and so on. Finally, an example is presented to describe the solution methodology and to illustrate the usefulness and effectiveness of the proposed technique. As we will see, the results presented in this paper reveal that the method is very effective and convenient for conservative nonlinear oscillators for which the restoring force has a nonpolynomial form. 


\section{Formulation and Solution Approach}

Consider a single-degree-of-freedom, conservative nonlinear oscillator governed by the dimensionless differential equation

$$
\frac{\mathrm{d}^{2} x}{\mathrm{~d} t^{2}}+f(x)=0
$$

with initial conditions

$$
x(0)=A \text { and } \frac{\mathrm{d} x}{\mathrm{~d} t}(0)=0,
$$

where the nonlinear function $f(x)$ is odd, i. e. $f(-x)=$ $-f(x)$, and satisfies $x f(-x)>0$ for $x \in[-A, A], x \neq 0$. Under these conditions $x=0$ is the equilibrium position. This is a conservative system that oscillates between the symmetric bounds $[-A, A]$ and, in general, the frequency and corresponding periodic solution are dependent on the oscillation amplitude $A$.

There exists no small parameter in (1) and therefore, the traditional perturbation methods cannot be applied directly. Due to the fact that the homotopy perturbation method requires neither a small parameter nor a linear term in a differential equation, we can approximately solve (1) using the homotopy perturbation method. In this method, an artificial perturbation equation is constructed by embedding an artificial parameter $p \in[0,1]$, which is used as expanding parameter. This technique yields a very rapid convergence of the solution series; in most cases, only one iteration leads to high accuracy of the solution.

In order to use the homotopy perturbation method, we re-write (1) in the form

$$
\frac{\mathrm{d}^{2} x}{\mathrm{~d} t}+x=x-f(x) .
$$

For (3) we can establish the following homotopy:

$$
\frac{\mathrm{d}^{2} x}{\mathrm{~d} t^{2}}+x=p(x-f(x)),
$$

where $p$ is the homotopy parameter. When $p=0$, (4) becomes a linear differential equation for which an exact solution can be calculated; for $p=1$, (4) becomes the original problem. Now the homotopy parameter $p$ is used to expand the solution $x(t)$ and the square of the unknown angular frequency $\omega$ as follows:

$$
\begin{aligned}
& x(t)=x_{0}(t)+p x_{1}(t)+p^{2} x_{2}(t)+\ldots, \\
& 1=\omega^{2}-p \alpha_{1}-p^{2} \alpha_{2}-\ldots,
\end{aligned}
$$

where $\alpha_{i}(i=1,2, \ldots)$ are to be determined. As can be seen, in (6) we use the parameter-expansion technology, which was first suggested by J. H. He [36]. In [14] Zhang and $\mathrm{Xu}$ systematically illustrated the solution procedure.

Substituting (5) and (6) into (4),

$$
\begin{aligned}
& \left(x_{0}^{\prime \prime}+p x_{1}^{\prime \prime}+p^{2} x_{2}^{\prime \prime}+\ldots\right) \\
& +\left(\omega^{2}-p \alpha_{1}-p^{2} \alpha_{2}-\ldots\right)\left(x_{0}+p x_{1}+p^{2} x_{2}+\ldots\right) \\
& =p\left[\left(x_{0}+p x_{1}+p^{2} x_{2}+\ldots\right)-f\left(x_{0}+p x_{1}+p^{2} x_{2}+\ldots\right)\right]
\end{aligned}
$$

and collecting the terms of the same power of $p$, we obtain a series of linear equations, of which we write only the first four:

$$
\begin{aligned}
& x_{0}^{\prime \prime}+\omega^{2} x_{0}=0, \quad x_{0}(0)=A, x_{0}^{\prime}(0)=0, \\
& x_{1}^{\prime \prime}+\omega^{2} x_{1}=\left(1+\alpha_{1}\right) x_{0}-f\left(x_{0}\right), \\
& x_{1}(0)=x_{1}^{\prime}(0)=0, \\
& x_{2}^{\prime \prime}+\omega^{2} x_{2}=\alpha_{2} x_{0}+\left(1+\alpha_{1}\right) x_{1}-x_{1} f^{\prime}\left(x_{0}\right), \\
& x_{2}(0)=x_{2}^{\prime}(0)=0, \\
& x_{3}^{\prime \prime}+\omega^{2} x_{3}=\alpha_{3} x_{0}+\left(1+\alpha_{2}\right) x_{1}+\alpha_{1} x_{2} \\
& \quad-x_{2} f^{\prime}\left(x_{0}\right)-\frac{1}{2} x_{1}^{2} f^{\prime \prime}\left(x_{0}\right), \\
& x_{3}(0)=x_{3}^{\prime}(0)=0 .
\end{aligned}
$$

In (8) - (10) we have taken into account the expression

$$
\begin{aligned}
f(x)= & f\left(x_{0}+p x_{1}+p^{2} x_{2}+p^{3} x_{3}+\ldots\right) \\
= & f\left(x_{0}\right)+p\left(\frac{\mathrm{d} f(x)}{\mathrm{d} x}\right)_{x=x_{0}} x_{1} \\
& +p^{2}\left[\left(\frac{\mathrm{d} f(x)}{\mathrm{d} x}\right)_{x=x_{0}} x_{2}+\frac{1}{2}\left(\frac{\mathrm{d}^{2} f(x)}{\mathrm{d} x^{2}}\right)_{x=x_{0}} x_{1}^{2}\right] \\
& +O\left(p^{3}\right)
\end{aligned}
$$

where

$$
f^{\prime}\left(x_{0}\right)=\left(\frac{\mathrm{d} f(x)}{\mathrm{d} x}\right)_{x=x_{0}}
$$

and

$$
f^{\prime \prime}\left(x_{0}\right)=\left(\frac{\mathrm{d}^{2} f(x)}{\mathrm{d} x^{2}}\right)_{x=x_{0}} .
$$

The solution of $(8)$ is

$$
x_{0}(t)=A \cos \omega t \text {. }
$$


Substitution of this result into the right-hand side of (9) gives

$$
x_{1}^{\prime \prime}+\omega^{2} x_{1}=\left(1+\alpha_{1}\right) A \cos \omega t-f(A \cos \omega t) .
$$

Since $f(x)$ is an odd function of $x, f(A \cos \omega t)$ can be expanded in a Fourier series as follows:

$$
\begin{aligned}
f(A \cos \omega t) & =\sum_{n=0}^{\infty} a_{2 n+1} \cos [(2 n+1) \omega t] \\
& =a_{1} \cos \omega t+a_{3} \cos 3 \omega t+\ldots,
\end{aligned}
$$

where

$$
\begin{aligned}
& a_{2 n+1}=\frac{4}{\pi} \int_{0}^{\pi / 2} f(A \cos \theta) \cos [(2 n+1) \theta] \mathrm{d} \theta, \\
& n=1,2, \ldots
\end{aligned}
$$

Substituting (17) into (16), we have

$$
\begin{aligned}
x_{1}^{\prime \prime}+\omega^{2} x_{1} & =\left[\left(1+\alpha_{1}\right) A-a_{1}\right] \cos \omega t \\
& -\sum_{n=1}^{\infty} a_{2 n+1} \cos [(2 n+1) \omega t] .
\end{aligned}
$$

No secular terms in $x_{1}(t)$ requires eliminating contributions proportional to $\cos \omega t$ on the right-hand side of (19), and setting the coefficient of $\cos \omega t$ to vanish yields

$$
\alpha_{1}=-1+\frac{a_{1}}{A} .
$$

From (6) and (20), writing $p=1$, we can easily find that the first approximation to the frequency of a nonlinear oscillator is

$$
\omega_{1}(A)=\sqrt{\frac{a_{1}}{A}},
$$

and the corresponding approximate periodic solution is given by (8).

Now in order to obtain the correction term $x_{1}$ for the periodic solution $x_{0}$ we consider the following procedure. Taking into account (20), we re-write (19) in the form

$$
x_{1}^{\prime \prime}+\omega^{2} x_{1}=-\sum_{n=1}^{\infty} a_{2 n+1} \cos [(2 n+1) \omega t]
$$

with the initial conditions $x_{1}(0)=0$ and $x_{1}^{\prime}(0)=0$. The periodic solution to (22) can be written as

$$
x_{1}(t)=\sum_{n=0}^{\infty} b_{2 n+1} \cos [(2 n+1) \omega t]
$$

Substituting (23) into (22) we obtain

$$
\begin{aligned}
& -\omega^{2} \sum_{n=0}^{\infty}(2 n+1)^{2} b_{2 n+1} \cos [(2 n+1) \omega t] \\
& +\omega^{2} \sum_{n=0}^{\infty} b_{2 n+1} \cos [(2 n+1) \omega t] \\
& =-\sum_{n=1}^{\infty} a_{2 n+1} \cos [(2 n+1) \omega t]
\end{aligned}
$$

which allows us to write the following expression for the coefficients $b_{2 n+1}$ :

$$
b_{2 n+1}=\frac{a_{2 n+1}}{4 n(n+1) \omega^{2}}
$$

for $n \geq 1$. Taking into account that $x_{1}(0)=0,(23)$ gives

$$
b_{1}=-\sum_{n=1}^{\infty} b_{2 n+1} \text {. }
$$

To determine the second-order approximate solution it is necessary to substitute (23) into (10). Then secular terms are eliminated and parameter $\alpha_{2}$ can be calculated. However, it is difficult to solve the new differential equation because $x_{1}(t)$ has an infinite number of harmonics [see (23)]. At this moment we introduce a modification in He's homotopy perturbation method to simplify the solution procedure. $x_{1}(t)$ has an infinite number of harmonics, however, we can truncate the series expansion at (23) and write an approximate equation $x_{1}^{(N)}(t)$ in the form

$$
x_{1}^{(N)}=b_{1}^{(N)} \cos \omega t+\sum_{n=1}^{N} b_{2 n+1} \cos [(2 n+1) \omega t],
$$

where

$$
b_{1}^{(N)}=-\sum_{n=1}^{N} b_{2 n+1} .
$$

This approximation is equivalent to consider the following approximation in (17):

$$
\begin{aligned}
f(A \cos \omega t) & \approx f^{(N)}(A \cos \omega t) \\
& =\sum_{n=0}^{N} a_{2 n+1} \cos [(2 n+1) \omega t] .
\end{aligned}
$$

Equation (27) has only a finite number of harmonics. Comparing (23) and (27), (26) and (28), and (17) and (29) it follows that

$$
\lim _{N \rightarrow \infty} x_{1}^{(N)}(t)=x_{1}(t)
$$




$$
\begin{aligned}
& \lim _{N \rightarrow \infty} b_{1}^{(N)}=b_{1}, \\
& \lim _{N \rightarrow \infty} f^{(N)}(A \cos \omega t)=f(A \cos \omega t) .
\end{aligned}
$$

In previous papers $[37,38]$ we considered the simplest case, $N=1(n=0,1)$, in (27)-(29), however, now we are going to improve He's homotopy perturbation method taking into account the approximation $N=2(n=0,1,2)$. We obtain

$$
\begin{aligned}
x_{1}^{(2)}(t)= & b_{3}(\cos 3 \omega t-\cos \omega t) \\
& +b_{5}(\cos 5 \omega t-\cos \omega t)
\end{aligned}
$$

and

$$
\begin{aligned}
f(A \cos \omega t) \approx & f^{(2)}(A \cos \omega t) \\
= & a_{1} \cos \omega t+a_{3} \cos 3 \omega t \\
& +a_{5} \cos 5 \omega t .
\end{aligned}
$$

From (25) the following expression for the coefficients $b_{3}$ and $b_{5}$ are obtained:

$$
\begin{aligned}
& b_{3}=\frac{a_{3}}{8 \omega^{2}}, \\
& b_{5}=\frac{a_{5}}{24 \omega^{2}} .
\end{aligned}
$$

Therefore, from (5), setting $p=1$, the first approximation to the periodic solution is given by

$$
\begin{aligned}
x_{a 1}(t)= & x_{0}(t)+x_{1}^{(2)}(t) \\
= & \left(A-b_{3}-b_{5}\right) \cos \omega_{1} t+b_{3} \cos 3 \omega_{1} t \\
& +b_{5} \cos 5 \omega_{1} t \\
= & \left(A-\frac{3 a_{3}+a_{5}}{24 \omega^{2}}\right) \cos \omega_{1} t+\frac{a_{3}}{8 \omega^{2}} \cos 3 \omega_{1} t \\
& +\frac{a_{5}}{24 \omega^{2}} \cos 5 \omega_{1} t
\end{aligned}
$$

where $\omega_{1}(A)$ is given by (21).

Substituting (15), (20) and (33) into (10) gives the following equation for $x_{2}(t)$ :

$$
\begin{aligned}
x_{2}^{\prime \prime}+ & \omega^{2} x_{2}=\alpha_{2} A \cos \omega t+\frac{a_{1} a_{3}}{8 A \omega^{2}}(\cos 3 \omega t-\cos \omega t) \\
& -\frac{a_{3}}{8 \omega^{2}} f^{\prime}(A \cos \omega t)(\cos 3 \omega t-\cos \omega t) \\
& -\frac{a_{5}}{24 \omega^{2}} f^{\prime}(A \cos \omega t)(\cos 5 \omega t-\cos \omega t)
\end{aligned}
$$

It is possible to do the following Fourier series expansions:

$$
\begin{gathered}
f^{\prime}(A \cos \omega t) \cos \omega t=\sum_{n=0}^{\infty} c_{2 n+1} \cos [(2 n+1) \omega t] \\
=c_{1} \cos \omega t+c_{3} \cos 3 \omega t+\ldots, \\
f^{\prime}(A \cos \omega t) \cos 3 \omega t=\sum_{n=0}^{\infty} d_{2 n+1} \cos [(2 n+1) \omega t] \\
=d_{1} \cos \omega t+d_{3} \cos 3 \omega t+\ldots, \\
f^{\prime}(A \cos \omega t) \cos 5 \omega t=\sum_{n=0}^{\infty} e_{2 n+1} \cos [(2 n+1) \omega t] \\
=e_{1} \cos \omega t+e_{3} \cos 3 \omega t+\ldots,
\end{gathered}
$$

where

$c_{2 n+1}=\frac{4}{\pi} \int_{0}^{\pi / 2} f^{\prime}(A \cos \theta) \cos \theta \cos [(2 n+1) \theta] \mathrm{d} \theta$,

$$
\begin{aligned}
& d_{2 n+1}=\frac{4}{\pi} \int_{0}^{\pi / 2} f^{\prime}(A \cos \theta) \cos 3 \theta \cos [(2 n+1) \theta] \mathrm{d} \theta, \\
& e_{2 n+1}=\frac{4}{\pi} \int_{0}^{\pi / 2} f^{\prime}(A \cos \theta) \cos 5 \theta \cos [(2 n+1) \theta] \mathrm{d} \theta .
\end{aligned}
$$

The secular term in the solution for $x_{2}(t)$ can be eliminated if

$$
\begin{aligned}
& \alpha_{2} A-\frac{a_{1} a_{3}}{8 A \omega^{2}}-\frac{a_{1} a_{5}}{24 A \omega^{2}} \\
& +\frac{\left(3 a_{3}+a_{5}\right) c_{1}}{24 \omega^{2}}-\frac{a_{3} d_{1}}{8 \omega^{2}}-\frac{a_{5} d_{1}}{24 \omega^{2}}=0 .
\end{aligned}
$$

Equation (45) can be solved for $\alpha_{2}$ :

$$
\begin{aligned}
\alpha_{2}=[ & a_{1}\left(3 a_{3}+a_{5}\right)-A\left(3 a_{3} c_{1}+a_{5} c_{1}\right. \\
& \left.\left.-3 a_{3} d_{1}-a_{5} e_{1}\right)\right]\left[24 A^{2} \omega_{2}\right]^{-1} .
\end{aligned}
$$

From (6), (20) and (45), and taking $p=1$, one can easily obtain the following expression for the secondorder approximate frequency:

$$
\begin{aligned}
\omega_{2}(A)= & \frac{1}{\sqrt{12 A}}\left\{6 a_{1}+\sqrt{6}\left[6 a_{1}^{2}+a_{1}\left(3 a_{3}+a_{5}\right)\right.\right. \\
& \left.\left.-A\left(3 a_{3} c_{1}+a_{5} c_{1}-3 a_{3} d_{1}-a_{5} e_{1}\right)\right]^{\frac{1}{2}}\right\}^{\frac{1}{2}} .
\end{aligned}
$$


With the requirement of (46), we can re-write (37) in the form

$$
\begin{aligned}
x_{2}^{\prime \prime}+\omega^{2} x_{2}= & \frac{a_{1} a_{3}}{8 A \omega^{2}} \cos 3 \omega t+\frac{a_{1} a_{5}}{24 A \omega^{2}} \cos 5 \omega t \\
& +\frac{3 a_{3}+a_{5}}{24 \omega^{2}} \sum_{n=1}^{\infty} c_{2 n+1} \cos [(2 n+1) \omega t] \\
& -\frac{a_{3}}{8 \omega^{2}} \sum_{n=1}^{\infty} d_{2 n+1} \cos [(2 n+1) \omega t] \\
& -\frac{a_{5}}{24 \omega^{2}} \sum_{n=1}^{\infty} e_{2 n+1} \cos [(2 n+1) \omega t]
\end{aligned}
$$

with the initial conditions $x_{2}(0)=0$ and $x_{2}^{\prime}(0)=0$. The solution of this equation is

$$
x_{2}(t)=\sum_{n=0}^{\infty} h_{2 n+1} \cos [(2 n+1) \omega t] .
$$

Substituting (49) into (48) we obtain

$$
\begin{gathered}
-\omega^{2} \sum_{n=0}^{\infty} 4 n(n+1) h_{2 n+1} \cos [(2 n+1) \omega t] \\
=\frac{a_{1} a_{3}}{8 A \omega^{2}} \cos 3 \omega t+\frac{a_{1} a_{5}}{24 A \omega^{2}} \cos 5 \omega t \\
+\frac{3 a_{3}+a_{5}}{24 \omega^{2}} \sum_{n=1}^{\infty} c_{2 n+1} \cos [(2 n+1) \omega t] \\
-\frac{a_{3}}{8 \omega^{2}} \sum_{n=1}^{\infty} d_{2 n+1} \cos [(2 n+1) \omega t] \\
-\frac{a_{5}}{24 \omega^{2}} \sum_{n=1}^{\infty} e_{2 n+1} \cos [(2 n+1) \omega t],
\end{gathered}
$$

where the coefficients are

$$
\begin{aligned}
& h_{3}=-\frac{3 a_{1} a_{3}+A\left(3 a_{3} c_{3}+a_{5} c_{3}-3 a_{3} d_{3}-a_{5} e_{3}\right)}{192 A \omega^{4}}, \\
& h_{5}=-\frac{a_{1} a_{5}+A\left[3 a_{3}\left(c_{5}-d_{5}\right)+a_{5}\left(c_{5}-e_{5}\right)\right]}{576 A \omega^{4}}, \\
& h_{2 n+1}=\frac{-\left(3 a_{3}+a_{5}\right) c_{2 n+1}+3 a_{3} d_{2 n+1}+a_{5} e_{2 n+1}}{96 n(n+1) \omega^{4}}, \\
& n \geq 3 .
\end{aligned}
$$

Truncating the infinite series at (49), it is possible to obtain the following second-order approximate solution for $x_{2}$ :

$$
x_{2}^{(N)}(t)=\sum_{n=0}^{N} h_{2 n+1} \cos [(2 n+1) \omega t],
$$

which has only a finite number of harmonics. As we are analyzing the second-order approximation we consider
$N=3$ in (54), in other words, only four harmonics ( $n=$ $0,1,2,3)$. In this situation, it is easy to verify that

$$
h_{1}=-h_{3}-h_{5}-h_{7}
$$

and

$$
h_{7}=\frac{-\left(3 a_{3}+a_{5}\right) c_{7}+3 a_{3} d_{7}+a_{5} e_{7}}{1152 A \omega^{4}} .
$$

Taking this into account, the approximate solution can be written as

$$
\begin{aligned}
x_{a 2}(t)= & x_{0}(t)+x_{1}^{(2)}(t)+x_{2}^{(3)}(t) \\
= & \left(A-b_{3}-b_{5}-h_{3}-h_{5}-h_{7}\right) \cos \omega_{2} t \\
& +\left(b_{3}+h_{3}\right) \cos 3 \omega_{2} t+\left(b_{5}+h_{5}\right) \cos 5 \omega_{2} t \\
& +h_{7} \cos 7 \omega_{2} t .
\end{aligned}
$$

\section{Illustrative Example: Oscillator with Fractional-Power Restoring Force}

In this section, we present an example to illustrate the usefulness and effectiveness of the proposed technique. The oscillator with restoring force expressed by fractional power is governed by the differential equation $[39,40]$

$$
\frac{\mathrm{d}^{2} x}{\mathrm{~d} t^{2}}+x^{1 / 3}=0
$$

with the initial conditions (2). For this oscillator we have $f(x)=x^{1 / 3}$ and $f^{\prime}(x)=\frac{1}{3} x^{-2 / 3}$. The Fourier series expansion of $f(A \cos \omega t)=A^{1 / 3} \cos ^{1 / 3} \omega t$ is given by (17), where

$$
\begin{aligned}
& a_{1}=\frac{3 A^{1 / 3} \Gamma(7 / 6)}{\sqrt{\pi} \Gamma(2 / 3)}, \\
& a_{3}=-\frac{3 A^{1 / 3} \Gamma(7 / 6)}{5 \sqrt{\pi} \Gamma(2 / 3)}=-\frac{1}{5} a_{1}, \\
& a_{5}=\frac{3 A^{1 / 3} \Gamma(7 / 6)}{10 \sqrt{\pi} \Gamma(2 / 3)}=\frac{1}{10} a_{1},
\end{aligned}
$$

and $\Gamma(z)$ is the Euler gamma function [41]. This integral and another that will appear later were solved using symbolic software such as Mathematica. From (21), the first analytical approximation for the frequency is

$$
\omega_{1}(A)=\sqrt{\frac{3 \Gamma(7 / 6)}{\sqrt{\pi} \Gamma(2 / 3) A^{2 / 3}}}=\frac{1.076851}{A^{1 / 3}} .
$$


For this example we have the following values for the Fourier coefficients:

$$
\begin{gathered}
c_{1}=\frac{\Gamma\left(\frac{7}{6}\right)}{\sqrt{\pi} \Gamma\left(\frac{2}{3}\right) A^{\frac{2}{3}}}, \quad c_{3}=-\frac{\Gamma\left(\frac{7}{6}\right)}{5 \sqrt{\pi} \Gamma\left(\frac{2}{3}\right) A^{\frac{2}{3}}}, \\
c_{5}=\frac{\Gamma(7 / 6)}{\sqrt{\pi} \Gamma\left(\frac{2}{3}\right) A^{\frac{2}{3}}}, \quad c_{7}=-\frac{7 \Gamma\left(\frac{7}{6}\right)}{110 \sqrt{\pi} \Gamma\left(\frac{2}{3}\right) A^{\frac{2}{3}}}, \\
d_{1}=-\frac{\Gamma\left(\frac{7}{6}\right)}{5 \sqrt{\pi} G\left(\frac{2}{3}\right) A^{\frac{2}{3}}}, \quad d_{3}=\frac{13 \Gamma\left(\frac{7}{6}\right)}{10 \sqrt{\pi} \Gamma\left(\frac{2}{3}\right) A^{\frac{2}{3}}}, \\
d_{5}=-\frac{20 \Gamma\left(\frac{7}{6}\right)}{11 \sqrt{\pi} \Gamma\left(\frac{2}{3}\right) A^{\frac{2}{3}}}, \quad d_{7}=\frac{2576 \Gamma\left(\frac{7}{6}\right)}{243 \sqrt{\pi} \Gamma\left(\frac{17}{3}\right) A^{\frac{2}{3}}}, \\
e_{1}=\frac{\Gamma\left(\frac{7}{6}\right)}{10 \sqrt{\pi} \Gamma\left(\frac{2}{3}\right) A^{\frac{2}{3}}}, \quad e_{3}=-\frac{4 \Gamma\left(\frac{7}{6}\right)}{11 \sqrt{\pi} \Gamma\left(\frac{2}{3}\right) A^{\frac{2}{3}}}, \\
e_{5}=\frac{31 \Gamma\left(\frac{7}{6}\right)}{22 \sqrt{\pi} \Gamma\left(\frac{2}{3}\right) A^{\frac{2}{3}}}, \quad e_{7}=-\frac{92960 \Gamma\left(\frac{7}{6}\right)}{729 \sqrt{\pi} \Gamma\left(\frac{20}{3}\right) A^{\frac{2}{3}}} .
\end{gathered}
$$

From (47), the second-order analytical approximation for the frequency is

$$
\begin{aligned}
\omega_{2}(A) & =\frac{1}{2 \pi^{\frac{1}{4}}} \sqrt{\frac{(60+\sqrt{3426}) \Gamma\left(\frac{7}{6}\right)}{10 \Gamma\left(\frac{2}{3}\right) A^{\frac{2}{3}}}} \\
& =\frac{1.070238}{A^{\frac{1}{3}}} .
\end{aligned}
$$

We illustrate the accuracy of the modified approach presented in this paper by comparing the approximate solutions previously obtained with the exact frequency $\omega_{\mathrm{ex}}$ and other results in the literature. In particular, we will consider the solution of (1) using the harmonic balance method. This method is a procedure for determining analytical approximations to the periodic solutions of differential equations using a truncated Fourier series representation. Like the homotopy perturbation method, the harmonic balance method can be applied to nonlinear oscillatory problems where a linear term does not exist, the nonlinear terms are not small, and there is no perturbation parameter.

The exact frequency is given by the expression (see Appendix)

$$
\omega_{\mathrm{ex}}(A)=\frac{1.070451}{A^{1 / 3}} .
$$

Applying the first-order and second-order approximations based on the exact harmonic balance method to the equation

$$
\left(\frac{\mathrm{d}^{2} x}{\mathrm{~d} t^{2}}\right)^{3}+x=0,
$$

Mickens achieved the following expressions for the frequency $[39,40]$ :

$$
\begin{aligned}
& \omega_{\mathrm{M} 1}(A)=\frac{1.04912}{A^{1 / 3}}, \text { relative error }=2.0 \%, \\
& \omega_{\mathrm{M} 2}(A)=\frac{1.06341}{A^{1 / 3}}, \text { relative error }=0.7 \% .
\end{aligned}
$$

Lim and Wu approximately solved (1) by using an improved harmonic balance method in which linearization is carried out prior to harmonic balancing [42]. They achieved the following results for the first-order and the second-order approximations:

$$
\begin{gathered}
\omega_{\mathrm{LW} 1}(A)=\frac{1.07685}{A^{1 / 3}}, \text { relative error }=0.6 \%, \\
\omega_{\mathrm{LW} 2}(A)=\frac{1.06928}{A^{1 / 3}}, \text { relative error }=0.11 \% .
\end{gathered}
$$

Finally, Wu, Sun and Lim [10] also approximately solved (1) using another improved harmonic balance method that incorporates salient features of both Newton's method and the harmonic balance method. They achieved the following results for the first-order, second-order and third-order approximations:

$$
\begin{gathered}
\omega_{\mathrm{WSL} 1}(A)=\frac{1.07685}{A^{1 / 3}}, \text { relative error }=0.6 \%, \\
\omega_{\mathrm{WSL} 2}(A)=\frac{1.06922}{A^{1 / 3}}, \text { relative error }=0.12 \%, \\
\omega_{\mathrm{WSL} 3}(A)=\frac{1.07078}{A^{1 / 3}}, \text { relative error }=0.031 \% .
\end{gathered}
$$

The frequency values for the first, second and third approximation orders and their relative errors obtained by Beléndez et al. [37] using a modified He's homotopy perturbation method [taking $N=1$ in (27) and (29), only two harmonics] are the following:

$$
\begin{gathered}
\omega_{\mathrm{B} 1}(A)=\frac{1.07685}{A^{1 / 3}}, \text { relative error }=0.6 \%, \\
\omega_{\mathrm{B} 2}(A)=\frac{1.06861}{A^{1 / 3}}, \text { relative error }=0.17 \%,
\end{gathered}
$$




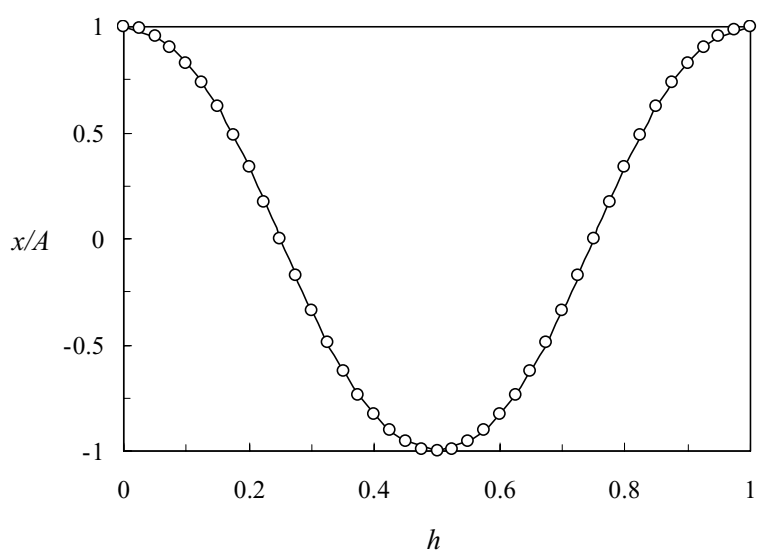

Fig. 1. Comparison of the approximate second-order periodic normalized solution (57) by the homotopy perturbation method (circles) with the exact solution (continuous line).

$$
\omega_{\mathrm{B} 3}(A)=\frac{1.07019}{A^{1 / 3}}, \text { relative error }=0.024 \% .
$$

The frequency values and their relative errors obtained in this paper applying an improved modified He's homotopy perturbation method are as follows:

$$
\begin{gathered}
\omega_{1}(A)=\frac{1.07685}{A^{1 / 3}}, \text { relative error }=0.6 \%, \\
\omega_{2}(A)=\frac{1.070238}{A^{1 / 3}}, \text { relative error }=0.020 \% .
\end{gathered}
$$

The accuracy $0.020 \%$ is remarkable good. In (70)(81), the percentage errors were evaluated using the equation

$$
\text { relative error of } \omega_{j}(\%)=100\left|\frac{\omega_{j}-\omega_{\mathrm{ex}}}{\omega_{\mathrm{ex}}}\right| .
$$

It is clear that at the second approximation order, the results obtained in this paper are better than those obtained previously by other authors. Besides, the second-order approximate frequency obtained using the improved modified perturbation method is better not only than the second-order approximate frequency obtained using other methods, but also than the third-order approximate frequencies. This example illustrates excellent accuracy of the analytical approximate frequencies.

The normalized exact periodic solution $x_{\mathrm{ex}} / A$ achieved by numerically integrating (58) and (2), and the proposed second-order normalized approximate periodic solution, $x_{a 2} / A$ in (57), are plotted in Figure 1. In this figure the parameter $h$ is defined as

$$
h=\frac{\omega_{\mathrm{ex}}(A)}{2 \pi} t \text {. }
$$

Note that this figure is valid for all values of $A$. It can be observed that the second-order approximate solution (57) gives excellent analytical approximate periodic solutions.

\section{Conclusions}

An improved He's homotopy perturbation method has been used to obtain the second-order approximate frequency for conservative nonlinear oscillators for which the elastic force is an odd nonlinear function. Excellent agreement between approximate frequencies and the exact one has been demonstrated and discussed, and the discrepancy of the second-order approximate frequency, $\omega_{2}(A)$, with respect to the exact one is as low as $0.0420 \%$. The general conclusion is that this improved modified homotopy perturbation method provides an easy and direct procedure for determining approximations to the periodic solutions of (1). An example has been presented to illustrate the excellent accuracy of the analytical frequency. This procedure also gives a very accurate estimate for the angular frequency at the second level of approximation. Finally, we think that the method has great potential and can be applied to other strongly nonlinear oscillators with nonpolynomial terms.

\section{Appendix}

Calculation of the exact angular frequency, $\omega_{\mathrm{ex}}(A)$, proceeds as follows. Integrating (54) and using the initial conditions (2), we arrive at

$$
\frac{1}{2}\left(\frac{\mathrm{d} x}{\mathrm{~d} t}\right)^{2}+\frac{3}{4} x^{4 / 3}=\frac{3}{4} A^{4 / 3} .
$$

From the representation above, we can derive the exact period as

$$
T_{\mathrm{ex}}(A)=\sqrt{\frac{32}{3}} \int_{0}^{A} \frac{\mathrm{d} x}{\sqrt{A^{4 / 3}-x^{4 / 3}}} .
$$

The substitution $x=A u^{3 / 4}$ gives, after some simplifications,

$$
\begin{aligned}
T_{\mathrm{ex}}(A) & =\sqrt{6} A^{1 / 3} \int_{0}^{1} u^{-1 / 4}(1-u)^{-1 / 2} \mathrm{~d} u \\
& =\sqrt{6} A^{1 / 3} B(3 / 4,1 / 2),
\end{aligned}
$$


where $B(m, n)$ is the Euler beta function [43]. Equation (A3) can be finally written as

$$
T_{\mathrm{ex}}(A)=\sqrt{6} A^{1 / 3} \frac{\Gamma(3 / 4) \Gamma(1 / 2)}{\Gamma(5 / 4)},
$$

where $\Gamma(z)$ is the Euler gamma function [41] and the following relation has been taken into account:

$$
B(m, n)=\frac{\Gamma(m) \Gamma(n)}{\Gamma(m+n)} .
$$

[1] J. H. He, J. Sound Vib. 229, 1257 (2000).

[2] J. H. He, Int. J. Non-linear Sci. Numer. Simulation 2, 317 (2001).

[3] T. Özis and A. Yildirim, J. Sound Vib. 301, 415 (2007).

[4] P. Amore, A. Raya, and F. M. Fernández, Eur. J. Phys. 26, 1057 (2005)

[5] R. E. Mickens, Oscillations in Planar Dynamics Systems, World Scientific, Singapore 1996.

[6] A. Beléndez, A. Hernández, A. Márquez, T. Beléndez, and C. Neipp, Eur. J. Phys. 27, 539 (2006).

[7] A. Beléndez, A. Hernández, T. Beléndez, M.L. Álvarez, S. Gallego, M. Ortuño, and C. Neipp, J. Sound Vib. 302, 1018 (2007).

[8] A. Beléndez and C. Pascual, Phys. Lett. 371, 291 (2007).

[9] G. R. Itovich and J. L. Moiola, Chaos, Solitons and Fractals 27, 647 (2005).

[10] B. S. Wu, W. P. Sun, and C. W. Lim, Int. J. Non-linear Mech. 41, 766 (2006).

[11] D. H. Shou and J. H. He, Int. J. Non-linear Sci. Numer. Simulation 8, 121 (2007).

[12] L. Xu, J. Comput. Appl. Math. 207, 148 (2007).

[13] J. H. He, Chaos, Solitons and Fractals 34, 1430 (2007).

[14] L.-N. Zhang and L. Xu, Z. Naturforsch. 62a, 387 (2007).

[15] D. H. Shou and J. H. He, Phys. Lett. A 372, 233 (2008).

[16] M. Tatari and M. Dehghan, Phys. Scr. 74, 310 (2006).

[17] B. Batiha, M. S. M. Noorani, and I. Hashim, Appl. Math. Comput. 186, 1322 (2007).

[18] A. Beléndez, A. Hernández, T. Beléndez, A. Márquez, and C. Neipp, Int. J. Non-linear Sci. Numer. Simulation 8, 329 (2007).

[19] Z.-Y. Ma, Z. Naturforsch. 62a, 387 (2007).

[20] J.H. He, Non-perturbative methods for strongly nonlinear problems, Dissertation, De-Verlag im Internet $\mathrm{GmbH}$, Berlin 2006.

[21] J. H. He, Int. J. Mod. Phys. B 20, 1141 (2006).

[22] J. H. He, Appl. Math. Comp. 151, 287 (2004).
The exact frequency can be obtained as

$$
\begin{aligned}
\omega_{\mathrm{ex}}(A) & =\frac{2 \pi}{T_{\mathrm{ex}}(A)}=\frac{2 \pi \Gamma(5 / 4)}{\sqrt{6} \Gamma(3 / 4) \Gamma(1 / 2) A^{1 / 3}} \\
& =\frac{1.070451}{A^{1 / 3}} .
\end{aligned}
$$

\section{Acknowledgements}

This work was supported by the "Ministerio de Educación y Ciencia", Spain, under project FIS200505881-C02-02 and by the "Generalitat Valenciana", Spain, 2008.

[23] X. C. Cai, W. Y. Wu, and M. S. Li, Int. J. Non-linear Sci. Numer. Simulation 7, 109 (2006).

[24] L. Cveticanin, Chaos, Solitons and Fractals 30, 1221 (2006).

[25] S. Abbasbandy, Chaos, Solitons and Fractals 30, 1206 (2006).

[26] A. Beléndez, A. Hernández, T. Beléndez, and A. Márquez, Eur. J. Phys. 28, 93 (2007).

[27] A. Beléndez, A. Hernández, T. Beléndez, E. Fernández, M. L. Álvarez, and C. Neipp, Int. J. Non-linear Sci. Numer. Simulation 8, 79 (2007).

[28] D. D. Ganji and A. Sadighi, Int. J. Non-linear Sci. Numer. Simulation 7, 411 (2006).

[29] J. H. He, Phys. Lett. A 350, 87 (2006).

[30] M. S. H. Chowdhury and I. Hashim, Phys. Lett. A 368, 251 (2007).

[31] F. Shakeri and M. Dehghan, Phys. Scr. 75, 551 (2007).

[32] A. Beléndez, C. Pascual, A. Márquez, and D. I. Méndez, Int. J. Non-linear Sci. Numer. Simulation 8, 483 (2007).

[33] A. Beléndez, C. Pascual, D. I. Méndez, M. L. Álvarez, and C. Neipp, Int. J. Non-linear Sci. Numer. Simulation 8, 493 (2007).

[34] L.-N. Zhang and J. H. He, Math. Prob. Eng. 2006, 1 (2006).

[35] A. Ghorbani and J. Saberi-Nadjafi, Int. J. Non-linear Sci. Numer. Simulation 8, 229 (2007).

[36] J. H. He, Int. J. Non-linear Mech. 37, 309 (2002).

[37] A. Beléndez, C. Pascual, S. Gallego, M. Ortuño, and C. Neipp, Phys. Lett. A 371, 421 (2007).

[38] A. Beléndez, C. Pascual, E. Fernández, C. Neipp, and T. Beléndez, Phys. Scr. 77, 025004 (2008).

[39] R. E. Mickens, J. Sound Vib. 246, 375 (2001).

[40] R. E. Mickens, J. Sound Vib. 255, 789 (2002).

[41] M. Abramowitz and I. A. Stegun (Eds.), "Gamma (Factorial) Function" and "Incomplete Gamma Function", $\S 6.1$ and $\S 6.5$, in: Handbook of Mathematical Func- 
tions with Formulas, Graphs and Mathematical Tables, Dover, New York 1972, pp. 258 and 263.

[42] C. W. Lim and B.S. Wu, J. Sound Vib. 281, 1157 (2005).

[43] M. Abramowitz and I. A. Stegun (Eds.), "Beta Func- tion" and "Incomplete Beta Function", $\S 6.2$ and $\S 6.6$, in: Handbook of Mathematical Functions with Formulas, Graphs and Mathematical Tables, Dover, New York 1972, pp. 258 and 263. 